\title{
On lacunary double statistical convergence in locally solid Riesz spaces
}

\section{Ekrem Savas*}

\section{"Correspondence:}

ekremsavas@yahoo.com Department of Mathematics, Istanbul Commerce University, Üsküdar, Istanbul, Turkey

\begin{abstract}
The concept of statistical convergence is one of the most active areas of research in the field of summability. Most of the new summability methods have relation with this popular method. In this paper, we introduce the concept of double $\mathcal{I}_{\theta}$-statistical- $\tau$-convergence which is a more general idea of statistical convergence. We also investigate the ideas of double $\mathcal{I}_{\theta}$-statistical- $\tau$-boundedness and double $\mathcal{I}_{\theta}$-statistical- $\tau$-Cauchy condition of sequences in the framework of locally solid Riesz space endowed with a topology $\tau$ and investigate some of their consequences. MSC: 40G15; 40A35; 46A40

Keywords: ideal; filter; double $\mathcal{I}$-statistical- $\tau$-convergence; double $\mathcal{I}_{\theta}$-statistical- $\tau$-convergence; double $\mathcal{I}_{\theta}$-statistical- $\tau$-boundedness; double $\mathcal{I}_{\theta}$-statistical- $\tau$-Cauchy condition
\end{abstract}

\section{Introduction}

The notion of statistical convergence, which is an extension of the idea of usual convergence, was introduced by Fast [1], Steinhaus [2] independently in the same year 1951 and also by Schoenberg [3]. Its topological consequences were studied first by Fridy [4] and Šalát [5]. The notion has also been defined and studied in different steps, for example, in a locally convex space [6]; in topological groups [7, 8]; in probabilistic normed spaces [9, 10], in intuitionistic fuzzy normed spaces [11], in random 2-normed spaces [12]. In [13] Albayrak and Pehlivan studied this notion in locally solid Riesz spaces. Recently, Mohiuddine et al. [14] studied statistically convergent, statistically bounded and statistically Cauchy for double sequences in locally solid Riesz spaces. Also, in [15] Mohiuddine et al. introduced the concept of lacunary statistical convergence, lacunary statistically bounded and lacunary statistically Cauchy in the framework of locally solid Riesz spaces. Quite recently, Das and Savas [16] introduced the ideas of $\mathcal{I}_{\tau}$-convergence, $\mathcal{I}_{\tau}$-boundedness and $\mathcal{I}_{\tau}$-Cauchy condition of nets in a locally solid Riesz space.

The more general idea of lacunary statistical convergence was introduced by Fridy and Orhan in [17]. Subsequently, a lot of interesting investigations have been done on this convergence (see, for example, [18-21] where more references can be found).

The idea of statistical convergence was further extended to $\mathcal{I}$-convergence in [22] using the notion of ideals of $\mathbb{N}$ with many interesting consequences. More investigations in this direction and more applications of ideals can be found in [22-31] where many important references can be found.

( 2013 Savas; licensee Springer. This is an Open Access article distributed under the terms of the Creative Commons Attribution License (http://creativecommons.org/licenses/by/2.0), which permits unrestricted use, distribution, and reproduction in any medium, provided the original work is properly cited. 
Recently in $[24,30]$ we used ideals to introduce the concepts of $\mathcal{I}^{\lambda}$-statistical convergence and $\mathcal{I}$-lacunary-statistical convergence and investigated their properties.

The notion of a Riesz space was first introduced by Riesz [32] in 1928, and since then it has found several applications in measure theory, operator theory, optimization and also in economics (see [33]). It is well known that a topology on a vector space that makes the operations of addition and scalar multiplication continuous is called a linear topology and a vector space endowed with a linear topology is called a topological vector space. A Riesz space is an ordered vector space which is also a lattice endowed with a linear topology. Further, if it has a base consisting of solid sets at zero, then it is known as a locally solid Riesz space.

In this paper, we introduce the idea of $\mathcal{I}$-double lacunary statistical convergence in a locally solid Riesz space and study some of its properties by using the mathematical tools of the theory of topological vector spaces.

\section{Preliminaries}

We now recall the following basic facts from [22].

A family $\mathcal{I}$ of subsets of a non-empty set $X$ is said to be an ideal if (i) $A, B \in \mathcal{I}$ implies $A \cup B \in \mathcal{I}$, (ii) $A \in \mathcal{I}, B \subset A$ imply $B \in \mathcal{I}$. $\mathcal{I}$ is called non-trivial if $\mathcal{I} \neq\{\phi\}$ and $X \notin \mathcal{I}$. $\mathcal{I}$ is admissible if it contains all singletons. If $\mathcal{I}$ is a proper non-trivial ideal, then the family of sets $F(\mathcal{I})=\left\{M \subset X: M^{c} \in \mathcal{I}\right\}$ is a filter on $X$ (where $c$ stands for the complement). It is called the filter associated with the ideal $\mathcal{I}$.

We also recall some of the basic concepts of Riesz spaces.

Definition 2.1 Let $L$ be a real vector space and let $\leq$ be a partial order on this space. $L$ is said to be an ordered vector space if it satisfies the following properties:

(i) If $x, y \in L$ and $y \leq x$, then $y+z \leq x+z$ for each $z \in L$.

(ii) If $x, y \in L$ and $y \leq x$, then $\lambda y \leq \lambda x$ for each $\lambda \geq 0$.

If in addition $L$ is a lattice with respect to the partial ordering, then $L$ is said to be a Riesz space (or a vector lattice).

For an element $x$ of a Riesz space $L$, the positive part of $x$ is defined by $x^{+}=x \vee \theta$, the negative part of $x$ by $x^{-}=(-x) \vee \theta$ and the absolute value of $x$ by $|x|=x \vee(-x)$, where $\theta$ is the element zero of $L$.

A subset $\mathrm{S}$ of a Riesz space $L$ is said to be solid if $y \in S$ and $|x| \leq|y|$ imply $x \in S$.

A topology $\tau$ on a real vector space $L$ that makes the addition and scalar multiplication continuous is said to be a linear topology, that is, when the mappings

$$
\begin{aligned}
& (x, y) \rightarrow x+y \quad(\text { from }(L \times L, \tau \times \tau) \rightarrow(L, \tau)), \\
& (\lambda, x) \rightarrow \lambda x \quad(\text { from }(R \times L, \sigma \times \tau) \rightarrow(L, \tau))
\end{aligned}
$$

are continuous, where $\sigma$ is the usual topology on $R$. In this case, the pair $(L, \tau)$ is called a topological vector space.

Every linear topology $\tau$ on a vector space $L$ has a base $\mathcal{N}$ for the neighborhoods of $\theta$ satisfying the following properties:

(a) Each $V \in \mathcal{N}$ is a balanced set, that is, $\lambda x \in V$ holds for all $x \in V$ and every $\lambda \in R$ with $|\lambda| \leq 1$. 
(b) Each $V \in \mathcal{N}$ is an absorbing set, that is, for every $x \in L$, there exists a $\lambda>0$ such that $\lambda x \in V$.

(c) For each $V \in \mathcal{N}$, there exists some $W \in \mathcal{N}$ with $W+W \subset V$.

Definition 2.2 A linear topology $\tau$ on a Riesz space $L$ is said to be locally solid if $\tau$ has a base at zero consisting of solid sets. A locally solid Riesz space $(L, \tau)$ is a Riesz space $L$ equipped with a locally solid topology $\tau$.

$\mathcal{N}_{\text {sol }}$ will stand for a base at zero consisting of solid sets and satisfying the properties (a), (b) and (c) in a locally solid topology.

\section{Main results}

The notion of statistical convergence depends on the density of subsets of $\mathbf{N}$, the set of natural numbers. A subset $E$ of $\mathbf{N}$ is said to have density $\delta(E)$ if

$$
\delta(E)=\lim _{n \rightarrow \infty} \frac{1}{n} \sum_{k=1}^{n} \chi_{E}(k) \quad \text { exists. }
$$

Note that if $K \subset \mathbf{N}$ is a finite set, then $\delta(K)=0$, and for any set $K \subset \mathbf{N}, \delta\left(K^{C}\right)=1-\delta(K)$.

Definition 3.1 A sequence $x=\left(x_{k}\right)$ is said to be statistically convergent to $\ell$ if for every $\varepsilon>0$,

$$
\delta\left(\left\{k \in \mathbf{N}:\left|x_{k}-\ell\right| \geq \varepsilon\right\}\right)=0 .
$$

In another direction, a new type of convergence called lacunary statistical convergence was introduced in [17] as follows. A lacunary sequence is an increasing integer sequence $\theta=\left\{k_{r}\right\}_{r \in \mathbb{N} \cup\{0\}}$ such that $k_{0}=0$ and $h_{r}=k_{r}-k_{r-1} \rightarrow \infty$ as $r \rightarrow \infty$. Let $I_{r}=\left(k_{r-1}, k_{r}\right]$ and $q_{r}=\frac{k_{r}}{k_{r-1}}$. A sequence $\left(x_{k}\right)$ of real numbers is said to be lacunary statistically convergent to $L$ (or, $S_{\theta}$-convergent to $L$ ) if for any $\epsilon>0$,

$$
\lim _{r \rightarrow \infty} \frac{1}{h_{r}}\left|\left\{k \in I_{r}:\left|x_{k}-L\right| \geq \epsilon\right\}\right|=0,
$$

where $|A|$ denotes the cardinality of $A \subset \mathbb{N}$. In [17] the relation between lacunary statistical convergence and statistical convergence was established among other things.

We now have the following definitions.

Definition 3.2 (See $[22,25])$ Let $\mathcal{I} \subset 2^{\mathbb{N}}$ be a proper admissible ideal in $\mathbb{N}$. The sequence $\left(x_{k}\right)$ of elements of $\mathbb{R}$ is said to be $\mathcal{I}$-convergent to $L \in \mathbb{R}$ if for each $\epsilon>0$, the set $A(\epsilon)=\{n \in$ $\left.\mathbb{N}:\left|x_{k}-L\right| \geq \epsilon\right\} \in \mathcal{I}$. The class of all $\mathcal{I}$-statistically convergent sequences will be denoted by $S(\mathcal{I})$.

Definition 3.3 ([24]) Let $\theta$ be a lacunary sequence. A sequence $x=\left(x_{k}\right)$ is said to be $\mathcal{I}$ lacunary statistically convergent to $L$ or $S_{\theta}(I)$-convergent to $L$ if for any $\epsilon>0$ and $\delta>0$,

$$
\left\{r \in \mathbb{N}: \frac{1}{h_{r}}\left|\left\{k \in I_{r}:\left|x_{k}-L\right| \geq \epsilon\right\}\right| \geq \delta\right\} \in \mathcal{I} .
$$


In this case, we write $x_{k} \rightarrow L\left(S_{\theta}(\mathcal{I})\right)$. The class of all $\mathcal{I}$-lacunary statistically convergent sequences will be denoted by $S_{\theta}(\mathcal{I})$.

It can be checked, as in the case of statistically and lacunary statistically convergent sequences, that both $S(\mathcal{I})$ and $S_{\theta}(\mathcal{I})$ are linear subspaces of the space of all real sequences.

Remark 3.1 For $\mathcal{I}=\mathcal{I}_{\text {fin }}=\{A \subseteq \mathbf{N}: A$ is a finite subset $\}, \mathcal{I}-S_{\theta}$-convergence coincides with lacunary statistical convergence which is defined in [17].

Let $E \subseteq \mathbf{N} \times \mathbf{N}$ be a two-dimensional set of positive integers and let $E_{m, n}$ be the numbers of $(i, j)$ in $K$ such that $i \leq n$ and $j \leq m$. Then the lower asymptotic density $\delta_{2}(E)$ of $E$ is defined as follows:

$$
\liminf _{m, n} \frac{E_{m, n}}{m n}=\delta_{2}(K)
$$

In the case when the sequence $\left(\frac{E_{m, n}}{m n}\right)_{m, n=1,1}^{\infty, \infty}$ has a limit, we say that $E$ has a natural density and is defined as follows:

$$
\lim _{m, n} \frac{E_{m, n}}{m n}=\delta_{2}(E)
$$

For example, let $E=\left\{\left(i^{2}, j^{2}\right):(i, j) \in \mathbf{N} \times \mathbf{N}\right\}$. Then

$$
\delta_{2}(E)=\lim _{m, n} \frac{E_{m, n}}{m n} \leq \lim _{m, n} \frac{\sqrt{m} \sqrt{n}}{m n}=0
$$

(i.e., the set $E$ has double natural density zero).

Recently, Mursaleen and Edely [34] presented the notion of statistical convergence for a double sequence $x=\left(x_{k l}\right)$ as follows:

A real double sequence $x=\left(x_{k l}\right)$ is said to be statistically convergent to $L$ provided that for each $\epsilon>0$,

$$
\lim _{m, n} \frac{1}{m n} \mid\left\{(k, l): k \leq m \text { and } l \leq n,\left|x_{k, l}-L\right| \geq \epsilon\right\} \mid=0 .
$$

The double sequence $\theta_{r, s}=\left\{\left(k_{r}, l_{s}\right)\right\}$ is called double lacunary if there exist two increasing sequences of integers such that

$$
k_{0}=0, \quad h_{r}=k_{r}-k_{k-1} \rightarrow \infty \quad \text { as } r \rightarrow \infty
$$

and

$$
l_{0}=0, \quad \bar{h}_{s}=l_{s}-l_{s-1} \rightarrow \infty \quad \text { as } s \rightarrow \infty .
$$

Let us denote $k_{r, s}=k_{r} l_{s}, h_{r, s}=h_{r} \bar{h}_{s}$ and $\theta_{r, s}$ is determined by $I_{r, s}=\left\{(k, l): k_{r-1}<k \leq\right.$ $k_{r}$ and $\left.l_{s-1}<l \leq l_{s}\right\}$.

We have the following. 
Definition 3.4 Let $\left(x_{k l}\right)$ be a sequence in a locally solid Riesz space $(L, \tau)$. We say that $x$ is $\mathcal{I}_{\theta_{r, s}}$-statistically- $\tau$-convergent to $x_{0}$ if for every $\tau$-neighborhood $U$ of zero and for $\delta>0$,

$$
\left\{(r, s) \in \mathbb{N} \times \mathbb{N}: \frac{1}{h_{r s}}\left|\left\{(k, l) \in I_{r s}: x_{k l}-x_{0} \notin U\right\}\right| \geq \delta\right\} \in \mathcal{I} .
$$

In this case, we write $\mathcal{I}_{\theta_{r, s}}-s t_{\tau}-\lim x_{k l}=x_{0}\left(\right.$ or $x_{k l} \stackrel{\mathcal{I}_{\theta r, s}-s t_{\tau}}{\longrightarrow} x_{0}$ in brief).

Remark 3.2 For $\mathcal{I}=\mathcal{I}_{f i n}, \mathcal{I}_{\theta_{r, s}}$-statistical- $\tau$-convergence becomes double lacunary statistical $\tau$-convergence in a locally solid Riesz space.

Definition 3.5 Let $\left(x_{k l}\right)$ be a sequence in a locally solid Riesz space $(L, \tau)$. We say that $x$ is $\mathcal{I}_{\theta_{r s}}$-statistically- $\tau$-bounded if for every $\tau$-neighborhood $U$ of zero and $\delta>0$, there exists $\alpha>0$ such that

$$
\left\{(r, s) \in \mathbb{N} \times \mathbb{N}: \frac{1}{h_{r s}}\left|\left\{(k, l) \in I_{r s}: \alpha x_{k l} \notin U\right\}\right| \geq \delta\right\} \in \mathcal{I} .
$$

Definition 3.6 Let $\left(x_{k l}\right)$ be a sequence in a locally solid Riesz space $(L, \tau)$. We say that $x$ is $\mathcal{I}_{\theta r, s}$-statistically- $\tau$-Cauchy if for every $\tau$-neighborhood $U$ of zero and $\delta>0$, there exist $p, q \in \mathbb{N}$ such that

$$
\left\{(r, s) \in \mathbb{N} \times \mathbb{N}: \frac{1}{h_{r s}}\left|\left\{(k, l) \in I_{r s}: x_{k l}-x_{p q} \notin U\right\}\right| \geq \delta\right\} \in \mathcal{I} .
$$

Now we are ready to present some basic properties of this new convergence in a locally solid Riesz space.

Theorem 3.1 Let $(L, \tau)$ be a Hausdorff locally solid Riesz space, $x=\left(x_{k l}\right)$ and $y=\left(y_{k l}\right)$ be two sequences in $L$. Then the following hold:

(a) If $\mathcal{I}_{\theta_{r, s}}-s t_{\tau}-\lim x_{k l}=y_{0}$ and $\mathcal{I}_{\theta_{r, s}}-s t_{\tau}-\lim x_{k l}=z_{0}$, then $y_{0}=z_{0}$.

(b) If $\mathcal{I}_{\theta_{r, s}}-s t_{\tau}-\lim x_{k l}=x_{0}$, then $\mathcal{I}_{\theta_{r, s}}-s t_{\tau}-\lim \alpha x_{k l}=\alpha x_{0}$ for each $\alpha \in \mathbb{R}$.

(c) If $\mathcal{I}_{\theta_{r, s}}-s t_{\tau}-\lim x_{k l}=x_{0}$ and $\mathcal{I}_{\theta_{r, s}}-s t_{\tau}-\lim y_{k l}=y_{0}$, then $\mathcal{I}_{\theta_{r, s}}-s t_{\tau}-\lim \left(x_{k l}+y_{k l}\right)=x_{0}+y_{0}$.

Proof (a) Let $U$ be any $\tau$-neighborhood of zero. Then there exists a $V \in \mathcal{N}_{\text {sol }}$ such that $V \subset U$. Take a $W \in \mathcal{N}_{\text {sol }}$ such that $W+W \subset V$. Let $\delta=\frac{1}{5}$. Since $\mathcal{I}_{\theta_{r, s}}-s t_{\tau}-\lim x_{k l}=y_{0}$ and $I_{\theta_{r, s}}-s t_{\tau}-\lim x_{k l}=z_{0}$, we write

$$
K_{1}=\left\{(r, s) \in \mathbb{N} \times \mathbb{N}: \frac{1}{h_{r s}}\left|\left\{(k, l) \in I_{r s}: x_{k l}-y_{0} \notin W\right\}\right|<\delta\right\} \in F(\mathcal{I})
$$

and

$$
K_{2}=\left\{(r, s) \in \mathbb{N} \times \mathbb{N}: \frac{1}{h_{r s}}\left|\left\{(k, l) \in I_{r s}: x_{k l}-z_{0} \notin W\right\}\right|<\delta\right\} \in F(\mathcal{I}) .
$$

Then $K=K_{1} \cap K_{2} \in F(\mathcal{I})$ and for $r, s \in K$,

$$
\frac{1}{h_{r s}}\left|\left\{(k, l) \in I_{r s}: x_{k l}-y_{0} \notin W\right\}\right|<\delta,
$$


i.e.,

$$
\frac{1}{h_{r s}}\left|\left\{(k, l) \in I_{r s}: x_{k l}-y_{0} \in W\right\}\right|>1-\delta=\frac{4}{5} .
$$

Similarly,

$$
\frac{1}{h_{r s}}\left|\left\{(k, l) \in I_{r s}: x_{k l}-z_{0} \in W\right\}\right|>\frac{4}{5} .
$$

Now write that $\left\{(k, l) \in I_{r s}: x_{(k, l)}-y_{0} \in W\right\}$ and $\left\{(k, l) \in I_{r s}: x_{k l}-z_{0} \in W\right\}$ cannot be disjoint, for then we will have $\frac{1}{h_{r s}}\left|\left\{(k, l) \in I_{r s}\right\}\right|>\frac{8}{5}$, which is impossible. So, there is a $\left(k_{r}, l_{s}\right) \in I_{r s}$ for which

$$
x_{k_{r}, l_{s}}-y_{0} \in W \quad \text { and } \quad x_{k_{r}, l_{s}}-z_{0} \in W .
$$

Then

$$
x_{0}-z_{0}=y_{0}-x_{k_{r} l_{s}}+x_{k_{r} l_{s}}-z_{0} \in W+W \subset V \subset U .
$$

Thus $y_{0}-z_{0} \in U$ for every $\tau$-neighborhood $U$ of zero. Since $(L, \tau)$ is Hausdorff, the intersection of all $\tau$-neighborhoods of zero is the singleton $\left\{\theta_{r, s}\right\}$, and so $y_{0}-z_{0}=\theta$, i.e., $y_{0}=z_{0}$.

(b) Let $\mathcal{I}_{\theta_{r, s}}-s t_{\tau}-\lim x_{k}=x_{0}$ and let $U$ be an arbitrary $\tau$-neighborhood of zero. Choose $V \in \mathcal{N}_{\text {sol }}$ such that $V \subset U$. For any $1>\delta>0$,

$$
K=\left\{(r, s) \in \mathbb{N} \times \mathbb{N}: \frac{1}{h_{r s}}\left|\left\{(k, l) \in I_{r s}: x_{k l}-x_{0} \notin V\right\}\right|<\delta\right\} \in F(\mathcal{I}),
$$

i.e., $\forall r, s \in K$,

$$
\frac{1}{h_{r s}}\left|\left\{(k, l) \in I_{r s}: x_{k l}-x_{0} \in V\right\}\right|>1-\delta .
$$

First let $|\alpha| \leq 1$. Since $V$ is balanced, $x_{k l}-x_{0} \in V$ implies that $\alpha\left(x_{k l}-x_{0}\right) \in V$. Therefore

$$
\left\{(k, l) \in I_{r s}: \alpha x_{k l}-\alpha x_{0} \in V\right\} \supset\left\{(k, l) \in I_{r s}: x_{k l}-x_{0} \in V\right\}
$$

and so $\forall r, s \in K$,

$$
\frac{1}{h_{r s}}\left|\left\{(k, l) \in I_{r s}: \alpha x_{k l}-\alpha x_{0} \in V\right\}\right| \geq \frac{1}{h_{r s}}\left|\left\{(k, l) \in I_{r s}: x_{k l}-x_{0} \in V\right\}\right|>1-\delta,
$$

which implies that

$$
\left\{(r, s) \in \mathbb{N} \times \mathbb{N}: \frac{1}{h_{r s}}\left|\left\{(k, l) \in I_{r s}: \alpha x_{k l}-\alpha x_{0} \notin V\right\}\right|<\delta\right\} \supset K
$$

and finally

$$
\left\{(r, s) \in \mathbb{N} \times \mathbb{N}: \frac{1}{h_{r s}}\left|\left\{(k, l) \in I_{r s}: \alpha x_{k l}-\alpha x_{0} \notin V\right\}\right|<\delta\right\} \in F(\mathcal{I}) .
$$


If $|\alpha|>1$ and $[|\alpha|]$ is the smallest integer greater or equal to $|\alpha|$, choose $W \in \mathcal{N}_{\text {sol }}$ such that $[|\alpha|] W \subset V$. Again, for $1>\delta>0$, taking

$$
K=\left\{(r, s) \in \mathbb{N} \times \mathbb{N}: \frac{1}{h_{r s}}\left|\left\{(k, l) \in I_{r s}: x_{k l}-x_{0} \notin W\right\}\right|<\delta\right\} \in F(\mathcal{I})
$$

and in view of the fact that

$$
\left|\alpha x_{0}-\alpha x_{k l}\right|=|\alpha|\left|x_{0}-x_{k l}\right| \leq[|\alpha|]\left|x_{n m}-x_{0}\right| \in[|\alpha|] W \subset V \subset U m,
$$

which implies that $\alpha x_{0}-\alpha x_{k l} \in V \subset U$, proceeding as before, we conclude that

$$
\left\{(r, s) \in \mathbb{N} \times \mathbb{N}: \frac{1}{h_{r s}}\left|\left\{(k, l) \in I_{r s}: \alpha x_{k l}-\alpha x_{0} \notin U\right\}\right|<\delta\right\} \in F(\mathcal{I}) .
$$

This proves that $I_{\theta_{r, s}}-s t_{\tau}-\lim \alpha x_{k l}=\alpha x_{0}$.

(c) Let $U$ be an arbitrary $\tau$-neighborhood of zero. Then there are $V, W \in \mathcal{N}_{\text {sol }}$ such that $W+W \subset V \subset U$. Since $I_{\theta}-s t_{\tau}-\lim x_{k l}=x_{0}$ and $I_{\theta_{r, s}}-s t_{\tau}-\lim y_{k l}=y_{0}$, we get, for $0<\delta<1$,

$$
K_{1}=\left\{(r, s) \in \mathbb{N} \times \mathbb{N}: \frac{1}{h_{r s}}\left|\left\{(k, l) \in I_{r s}: x_{k l}-x_{0} \notin W\right\}\right|<\frac{\delta}{3}\right\} \in F(\mathcal{I})
$$

and

$$
K_{2}=\left\{(r, s) \in \mathbb{N} \times \mathbb{N}: \frac{1}{h_{r s}}\left|\left\{(k, l) \in I_{r s}: y_{k l}-y_{0} \notin W\right\}\right|<\frac{\delta}{3}\right\} \in F(\mathcal{I}) .
$$

If $K=K_{1} \cap K_{2}$, then $\forall r, s \in K$,

$$
\frac{1}{h_{r s}}\left|\left\{(k, l) \in I_{r s}: x_{k l}-x_{0} \notin W\right\}\right|<\frac{\delta}{3},
$$

i.e.,

$$
\frac{1}{h_{r s}}\left|\left\{(k, l) \in I_{r s}: x_{k l}-x_{0} \in W\right\}\right|>1-\frac{\delta}{3}
$$

and also

$$
\frac{1}{h_{r s}}\left|\left\{(k, l) \in I_{r s}: y_{k l}-y_{0} \notin W\right\}\right|<\frac{\delta}{3} .
$$

But

$$
\left(x_{k l}+y_{k l}\right)-\left(x_{0}+y_{0}\right)=\left(x_{k l}-x_{0}\right)+\left(y_{k l}-y_{0}\right) \in W+W \subset V \subset U
$$

$\forall(k, l) \in I_{r s}$ such that $k, l \in A \cap B$ when $\left\{(k, l) \in I_{r s}: x_{k l}-x_{0} \in W\right\}=A$ (say) and $\left\{(k, l) \in I_{r s}: y_{k l}-y_{0} \in W\right\}=B$ (say). Note that

$$
|A|=|A \cap B|+|A \backslash B| \leq|A \cap B|+\left|B^{c}\right|,
$$


i.e.,

$$
\begin{aligned}
\frac{1}{h_{r s}}|A| & \leq \frac{1}{h_{r s}}|A \cap B|+\frac{1}{h_{r s}}\left|B^{c}\right| \\
& <\frac{1}{h_{r s}}|A \cap B|+\frac{\delta}{3},
\end{aligned}
$$

i.e.,

$$
\begin{aligned}
\frac{1}{h_{r s}}|A \cap B| & =\frac{1}{h_{r s}}\left|\left\{(k, l) \in I_{r s}: x_{k l}-x_{0} \in W \wedge y_{k l}-y_{0} \in W\right\}\right| \\
& >\frac{1}{h_{r s}}\left|\left\{(k, l) \in I_{r s}: x_{k l}-x_{0} \in W\right\}\right|-\frac{\delta}{3} \\
& >1-\frac{\delta}{3}-\frac{\delta}{3} \\
& >1-\delta .
\end{aligned}
$$

Since

$$
\left\{(k, l) \in I_{r s}:\left(x_{k l}+y_{k l}\right)-\left(x_{0}+y_{0}\right) \in U\right\} \supset A \cap B,
$$

so for all $r, s \in K$,

$$
\frac{1}{h_{r s}}\left|\left\{(k, l) \in I_{r s}:\left(x_{k l}+y_{k l}\right)-\left(x_{0}+y_{0}\right) \in U\right\}\right| \geq \frac{1}{h_{r s}}|A \cap B|>1-\delta,
$$

i.e.,

$$
\frac{1}{h_{r s}}\left|\left\{(k, l) \in I_{r s}:\left(x_{k l}+y_{k l}\right)-\left(x_{0}+y_{0}\right) \notin U\right\}\right|<\delta .
$$

Hence

$$
K \subset\left\{(r, s) \in \mathbb{N} \times \mathbb{N}: \frac{1}{h_{r s}}\left|\left\{(k, l) \in I_{r s}:\left(x_{k l}+y_{k l}\right)-\left(x_{0}+y_{0}\right) \notin U\right\}\right|<\delta\right\}
$$

and so

$$
\left\{(r, s) \in \mathbb{N} \times \mathbb{N}: \frac{1}{h_{r s}}\left|\left\{(k, l) \in I_{r s}:\left(x_{k l}+y_{k l}\right)-\left(x_{0}+y_{0}\right) \in U\right\}\right|<\delta\right\} \in F(\mathcal{I}) .
$$

This completes the proof of the theorem.

Theorem 3.2 Let $(L, \tau)$ be a locally solid Riesz space. Let $x=\left\{x_{k l}\right\}, y=\left\{y_{k l}\right\}$ and $z=\left\{z_{k l}\right\}$ be three sequences in $L$ such that $x_{k l} \leq y_{k l} \leq z_{k l}$ for each $(k, l) \in \mathbb{N} \times \mathbb{N}$. If $\mathcal{I}_{\theta_{r, s}}-s t_{\tau}-\lim x_{k l}=$ $a=\mathcal{I}_{\theta_{r, s}}-s t_{\tau}-\lim z_{n m}$, then $\mathcal{I}_{\theta_{r, s}}-s t_{\tau}-\lim y_{k l}=a$.

Proof Let $U$ be an arbitrary $\tau$-neighborhood of zero. Take $V, W \in \mathcal{N}_{\text {sol }}$ such that $W+W \subset$ $V \subset U$. Since $\mathcal{I}_{\theta_{r, s}}-s t_{\tau}-\lim x_{k l}=a=\mathcal{I}_{\theta_{r, s}}-s t_{\tau}-\lim z_{k l}$, so for $0<\delta<1$,

$$
K_{1}=\left\{(r, s) \in \mathbb{N} \times \mathbb{N}: \frac{1}{h_{r s}}\left|\left\{(k, l) \in I_{r s}: x_{k l}-a \notin W\right\}\right|<\frac{\delta}{3}\right\} \in F(\mathcal{I})
$$


and

$$
K_{2}=\left\{(r, s) \in \mathbb{N} \times \mathbb{N}: \frac{1}{h_{r s}}\left|\left\{(k, l) \in I_{r s}: z_{k l}-a \notin W\right\}\right|<\frac{\delta}{3}\right\} \in F(\mathcal{I}) .
$$

Hence we observe that $\forall r, s \in K$,

$$
\frac{1}{h_{r s}}\left|\left\{(k, l) \in I_{r s}: x_{k l}-a \notin W\right\}\right|<\frac{\delta}{3},
$$

i.e.,

$$
\frac{1}{h_{r s}}\left|\left\{(k, l) \in I_{r s}: x_{k l}-a \in W\right\}\right|>1-\frac{\delta}{3}
$$

and

$$
\frac{1}{h_{r s}}\left|\left\{(k, l) \in I_{r s}: z_{k l}-a \notin W\right\}\right|<\frac{\delta}{3} .
$$

Writing $A=\left\{(k, l) \in I_{r s}: x_{k l}-a \in W\right\}$ and $B=\left\{(k, l) \in I_{r s}: z_{k l}-a \in W\right\}$, we see that $\forall k, l \in$ $A \cap B$,

$$
\begin{aligned}
& x_{k l} \leq y_{k l} \leq z_{k l}, \\
& x_{k l}-a \leq y_{k l}-a \leq z_{k l}-a, \\
& \left|y_{k l}-a\right| \leq\left|x_{k l}-a\right|+\left|z_{k l}-a\right| \in W+W \subset V,
\end{aligned}
$$

and as $V$ is solid, so

$$
y_{k l}-a \in V \subset U
$$

Clearly, $\left\{(k, l) \in I_{r s}: y_{k l}-a \in U\right\} \supset A \cap B$ and as in the previous theorem, we show that $\forall r, s \in K$,

$$
\frac{1}{h_{r s}}\left|\left\{(k, l) \in I_{r s}: y_{k l}-a \in U\right\}\right| \geq \frac{1}{h_{r s}}|A \cap B|>1-\delta,
$$

i.e.,

$$
\frac{1}{h_{r s}}\left|\left\{(k, l) \in I_{r s}: y_{k l}-a \notin U\right\}\right|<\delta .
$$

Hence

$$
\left\{(r, s) \in \mathbb{N} \times \mathbb{N}: \frac{1}{h_{r s}}\left|\left\{(k, l) \in I_{r s}: y_{k l}-a \notin U\right\}\right|<\delta\right\} \supset K,
$$

where $K \in F(\mathcal{I})$ and so

$$
\left\{(r, s) \in \mathbb{N} \times \mathbb{N}: \frac{1}{h_{r s}}\left|\left\{k \in I_{r s}: y_{k l}-a \notin U\right\}\right| \geq \delta\right\} \in \mathcal{I} .
$$

This proves that $\mathcal{I}_{\theta_{r, s}}-s t_{\tau}-\lim y_{k l}=a$. This completes the proof of the theorem. 
Theorem 3.3 An $\mathcal{I}_{\theta_{r, s}}$-statistically $\tau$-convergent sequence $\left(x_{k l}\right)$ in a locally solid Riesz space $(L, \tau)$ is $\mathcal{I}_{\theta r, s}$-statistically $\tau$-bounded.

Proof Let $\left(x_{k l}\right)$ be $I_{\theta_{r, s}}$-statistically $\tau$-convergent to $x_{0} \in L$. Let $\mathrm{U}$ be an arbitrary $\tau$ neighborhood of zero. Choose $V, W \in \mathcal{N}_{\text {sol }}$ such that $W+W \subset V \subset U$. Since $W$ is absorbing, there is a $\mu>0$ such that $\mu x_{0} \in W$. Choose $\alpha \leq 1$ so that $\alpha \leq \mu$. Since $W$ is solid and $\left|\lambda x_{0}\right| \leq\left|\mu x_{0}\right|$, we have $\alpha x_{0} \in W$. Again, as $W$ is balanced, $x_{k l}-x_{0} \in W$ implies that $\alpha\left(x_{n m}-x_{0}\right) \in W$. Now, for any $0<\delta<1$,

$$
K=\left\{(r, s) \in \mathbb{N} \times \mathbb{N}: \frac{1}{h_{r s}}\left|\left\{(k, l) \in I_{r s}: x_{k l}-x_{0} \notin W\right\}\right|<\delta\right\} \in F(\mathcal{I}) .
$$

Thus, for all $r, s \in K$,

$$
\frac{1}{h_{r s}}\left|\left\{(k, l) \in I_{r s}: x_{k l}-x_{0} \notin W\right\}\right|<\delta,
$$

i.e.,

$$
\frac{1}{h_{r s}}\left|\left\{(k, l) \in I_{r s}: x_{k l}-x_{0} \in W\right\}\right|>1-\delta .
$$

If $B_{r s}=\left\{(k, l) \in I_{r s}: x_{k l}-x_{0} \in W\right\}$, then $\forall k, l \in B_{r s}$

$$
\alpha x_{k l}=\alpha\left(x_{k l}-x_{0}\right)+\alpha x_{0} \in W+W \subset V \subset U,
$$

and so, for all $r, s \in K$,

$$
\begin{aligned}
\frac{1}{h_{r s}}\left|\left\{(k, l) \in I_{r s}: \alpha x_{k l} \in W\right\}\right| & \geq \frac{1}{h_{r s}}\left|\left\{(k, l) \in I_{r s}: x_{k l}-x_{0} \in W\right\}\right| \\
& >1-\delta
\end{aligned}
$$

i.e.,

$$
\frac{1}{h_{r s}}\left|\left\{(k, l) \in I_{r s}: \alpha x_{k l} \notin W\right\}\right|<\delta .
$$

Hence

$$
K \subset\left\{(r, s) \in \mathbb{N} \times \mathbb{N}: \frac{1}{h_{r s}}\left|\left\{(k, l) \in I_{r s}: \alpha x_{k l} \notin W\right\}\right|<\delta\right\} .
$$

Since $K \in F(\mathcal{I})$, so the set on the right-hand side also belongs to $F(\mathcal{I})$ and this proves that $\left(x_{k l}\right)$ is $\mathcal{I}_{\theta_{r, s}}$-statistically $\tau$-bounded.

Theorem 3.4 If a sequence $\left(x_{k l}\right)$ in a locally solid Riesz space $(L, \tau)$ is $\mathcal{I}_{\theta_{r, s}}$-statistically $\tau$-convergent, then it is $\mathcal{I}_{\theta r, s}$-statistically $\tau$-Cauchy.

Proof Let $\left(x_{k l}\right)$ be $\mathcal{I}_{\theta_{r, s}}$-statistically $\tau$-convergent to $x_{0} \in L$. Let $\mathrm{U}$ be an arbitrary $\tau$ neighborhood of zero. Choose $V, W \in \mathcal{N}_{\text {sol }}$ such that $W+W \subset V \subset U$. Let $0<\delta<1$. 
Therefore

$$
K=\left\{(r, s) \in \mathbb{N} \times \mathbb{N}: \frac{1}{h_{r s}}\left|\left\{(k, l) \in I_{r s}: x_{k l}-x_{0} \notin W\right\}\right|<\delta\right\} \in F(\mathcal{I}) .
$$

For all $r, s \in K$,

$$
\frac{1}{h_{r s}}\left|\left\{(k, l) \in I_{r s}: x_{k l}-x_{0} \notin W\right\}\right|<\delta,
$$

i.e.,

$$
\frac{1}{h_{r s}}\left|\left\{(k, l) \in I_{r s}: x_{k l}-x_{0} \in W\right\}\right|>1-\delta .
$$

Take $r, s \in K$ and in view of the above, we can choose $p, q \in\left\{(k, l) \in I_{r s}: x_{k l}-x_{0} \in\right.$ $W\}$ (since this set cannot be empty). Then $x_{p q}-x_{0} \in W$. Now observe that if for $(k, l) \in I_{r s}, x_{k l}-x_{0} \in W$, then

$$
x_{k l}-x_{p q}=x_{k l}-x_{0}+x_{0}-x_{p q} \in W+W \subset V \subset U
$$

Hence, as in the earlier proofs, we can prove that

$$
K \subset\left\{(r, s) \in \mathbb{N} \times \mathbb{N}: \frac{1}{h_{r s}}\left|\left\{(k, l) \in I_{r s}: x_{k l}-x_{p q} \notin W\right\}\right|<\delta\right\},
$$

which consequently implies that $\left(x_{k l}\right)$ is $\mathcal{I}_{\theta_{r, s}}$-statistically $\tau$-Cauchy.

This completes the proof of the theorem.

It should be noted that single and double case of $I_{\lambda}$-statistical convergence in locally solid Riesz spaces are introduced in [35] and [36] respectively.

\section{Competing interests}

The author declares that they have no competing interests.

Received: 24 October 2012 Accepted: 15 February 2013 Published: 13 March 2013

\section{References}

1. Fast, H: Sur la convergence statistique. Collog. Math. 2, 241-244 (1951)

2. Steinhaus, H: Sur la convergence ordinaire et la convergence asymptotique. Colloq. Math. 27, 73-74 (1951)

3. Schoenberg, IJ: The integrability of certain functions and related summability methods. Am. Math. Mon. 66, 361-375 (1959)

4. Fridy, JA: On statistical convergence. Analysis 5, 301-313 (1985)

5. Šalát, T: On statistically convergent sequences of real numbers. Math. Slovaca 30, 139-150 (1980)

6. Maddox, IJ: Statistical convergence in locally convex spaces. Math. Proc. Camb. Philos. Soc. 104, 141-145 (1988)

7. Cakalli, H: Statistical convergence in topological groups. Pure Appl. Math. Sci. 43(1-2), 27-31 (1996)

8. Cakalli, H, Savas, E: Statistical convergence of double sequences in topological groups. J. Comput. Anal. Appl. 12(2), $421-426(2010)$

9. Mohiuddine, SA, Savaş, E: Lacunary statistically convergent double sequences in probabilistic normed spaces. Ann. Univ. Ferrara, Sez. 7: Sci. Mat. 58(2), 331-339 (2012)

10. Savaş, E, Mohiuddine, SA: $\lambda$-statistically convergent double sequences in probabilistic normed space. Math. Slovaca 62(1), 99-108 (2012)

11. Mursaleen, M, Mohiuddine, SA: Statistical convergence of double sequences in intuitionistic fuzzy normed spaces. Chaos Solitons Fractals 41(5), 2414-2421 (2009)

12. Savaş, E: On generalized double statistical convergence in random 2-normed space. J. Inequal. Appl. 2012, Article ID 209 (2012)

13. Albayrak, H, Pehlivan, S: Statistical convergence and statistical continuity on locally solid Riesz spaces. Topol. Appl. 159, 1887-1893 (2012) 
14. Mohiuddine, SA, Alotaibi, A, Mursaleen, M: Statistical convergence of double sequences in locally solid Riesz spaces. Abstr. Appl. Anal. 2012, Article ID 719729 (2012)

15. Mohiuddine, SA, Alghamdi, MA: Statistical summability through lacunary sequence in locally solid Riesz spaces. J. Inequal. Appl. 2012, Article ID 225 (2012)

16. Das, P, Savaş, E: On I-convergence of nets in locally solid Riesz spaces. Filomat 27(1), 84-89 (2013)

17. Fridy, JA, Orhan, C: Lacunary statistical convergence. Pac. J. Math. 160, 43-51 (1993)

18. Patterson, RF, Savaş, E: Lacunary statistical convergence of double sequences. Math. Commun. 10, 55-61 (2000)

19. Savaş, E: On some new double lacunary sequences spaces via Orlicz function. J. Comput. Anal. Appl. 11(3), 423-430 (2009)

20. Savaş, E, Patterson, RF: Some double lacunary sequence spaces defined by Orlicz functions. Southeast Asian Bull. Math. 35(1), 103-110 (2011)

21. Savaş, E, Patterson, RF: Double $\sigma$-convergence lacunary statistical sequences. J. Comput. Anal. Appl. 11(4), 610-615 (2009)

22. Kostyrko, P, Šalát, T, Wilczynki, W: I-convergence. Real Anal. Exch. 26(2), 669-685 (2000/2001)

23. Dems, K: On I-Cauchy sequences. Real Anal. Exch. 30, 123-128 (2004/2005)

24. Das, P, Savaş, E, Ghosal, SK: On generalizations of certain summability methods using ideals. Appl. Math. Lett. 24, 1509-1514 (2011)

25. Kostyroko, P, Macaj, M, Šalát, T, Sleziak, M: I-convergence and extremal /-limit points. Math. Slovaca 55, 443-464 (2005)

26. Lahiri, BK, Das, P: On / and I* convergence of nets. Real Anal. Exch. 33(2), 431-442 (2007/2008)

27. Savaş, E: $\Delta^{m}$-strongly summable sequence spaces in 2-normed spaces defined by ideal convergence and an Orlicz function. Appl. Math. Comput. 217, 271-276 (2010)

28. Savaş, E: A-sequence spaces in 2-normed space defined by ideal convergence and an Orlicz function. Abstr. Appl. Anal. 2011, Article ID 741382 (2011)

29. Savaş, E: On some new sequence spaces in 2-normed spaces using ideal convergence and an Orlicz function. J. Inequal. Appl. 2010, Article ID 482392 (2010). doi:10.1155/2010/482392

30. Savaș, E, Das, P: A generalized statistical convergence via ideals. Appl. Math. Lett. 24, 826-830 (2011)

31. Savaş, E, Das, P, Dutta, S: A note on strong matrix summability via ideals. Appl. Math. Lett. 25(4), $733-738$ (2012)

32. Riesz, F: Sur la decomposition des operations functionelles lineaires. In: Alti del Congr. Internaz. del Mat., Bologna 1928, vol. 3, pp. 143-148. Zanichelli, Bologna (1930)

33. Aliprantis, CD, Burkinshaw, O: Locally Solid Riesz Spaces with Applications to Economics, 2nd edn. Am. Math. Soc. Providence (2003)

34. Mursaleen, M, Edely, OH: Statistical convergence of double sequences. J. Math. Anal. Appl. 288, $223-231$ (2003)

35. Das, P, Savaş, E: On $/ \lambda$-statistical convergence in locally solid Riesz spaces. Math. Slovaca. Preprint

36. Savas, E: On generalized double statistical convergence in locally solid Riesz spaces. Miskolc Math. Notes. Preprint

doi:10.1186/1029-242X-2013-99

Cite this article as: Savas: On lacunary double statistical convergence in locally solid Riesz spaces. Journal of Inequalities and Applications 2013 2013:99.

\section{Submit your manuscript to a SpringerOpen ${ }^{\circ}$ journal and benefit from:}

- Convenient online submission

Rigorous peer review

- Immediate publication on acceptance

- Open access: articles freely available online

- High visibility within the field

- Retaining the copyright to your article 